\title{
Commutators of two operators one of which is unbounded and semi-normal.
}

\author{
Mendel Davio (*)
}

\begin{abstract}
Summnxy. - The concept of semi-normality and hyponormality are defined for unbounded operators in Hilbert Space and Commutators $A B-B A$, with $A$ unbounded and seminormal and with $B$ bounded, are studied. Four theorems are proved. Tno of them are generalizations of results of C.R. Putman.
\end{abstract}

\section{1. - Introduction.}

In this paper, problems concerning commutators of (Linear not necessarily bounded) operators defined in a separable infinite dimensional Hilbert Space $H$ will be treated (**).

The domain of definition and the range of an operator $A$ will be denoted by $\mathscr{D}_{A}$ and $\mathscr{R}_{A}$, respectively. The fact that $B$ is an extension of $A$ will be denoted by $A$ c $B$. The bounded operators will be considered as defined on the whole space $H$.

We quote a theorem of C.R. Punnam concerning bounded operators.

(a) If $A$ is a semi-normal bounded operator and $B$ and $C$ are bounded operators such that

$$
A B-B A=C
$$

then

(see [2] p. 128).

$$
\inf _{\|x\|=1}|(C x, x)|=0
$$

In particular, this theorem is true when $A$ is hyponormal or normal.

We recall that a bounded operator $A$ is called hyponormal if $\left\|A^{*} x\right\| \leq$ $\leq\|A x\|$ an is ealled semi-normal when $A$ or $A^{*}$ is hyponormal. In section 2 , the notions of hyponormality and semi-normality for linear, not necessarily bounded, operators will be defined and an example of a not normal seminormal unbounded operator will be given.

In section 3 , theorem (a) will be extended to various cases in which $A$ and $C$ are not necessarily bounded. In Theorem 1 the case in which $A^{*}$ is

(*) Entrata in Redazione il 5 dicembre 1968.

(**) This paper forms part of a thesis in partial fulfilment of the requirements for the degree of Doctor of Science at the Technion.Israel Institute of Technology. The author wishes to thank Professor M. Ruchaw for his help in preparing the paper. 
hyponormal will be considered. Theorem 1 will be used in the proof of Theorem 2 which is a generalization of Theorem (a). The case in which $A$ is normal will be considered in Theorem 3.

Finally, Theorem 4 is a generalization of theorem 2.10 .1 in [3].

2. - Now we define the notions of hyponormality and semi-normality for not necessarily bounded operators.

Defintrion 1. - An operator $A$ is called hyponormal if it is closed, densely defined and satisfies the conditions

$$
\begin{gathered}
\mathfrak{D}_{A}=\mathfrak{D}_{A^{*}}, \\
\left\|A^{*} x\right\| \leq\|A x\| \text { for } x \in \mathfrak{D}_{A} .
\end{gathered}
$$

Definimion 2. - An operator $A$ is called semi-normal if it is closed, densely defined and if $A$ or $A^{*}$ is hyponormal.

It is clear that every normal operator is hyponormal and therefore seminormal. Moreover the above definitions are extensions of the definitions of hyponormality and semi-normality for bounded operators. The following is a simple example of a semi-normal unbounded operator which is not normal. Let $H=l_{2}$ and let $A$ be the infinite matrix.

$$
\left(\alpha_{i j}\right)_{i, j=1}^{\infty} \text { where }
$$

$$
a_{i, i+1}=i \text { for } i=1,2, \ldots \text { and } a_{i j}=0 \text { for } j \neq i+1
$$

We will show that $A^{*}$ is hyponormal. It is clear that $A$ is closed and densely defined. It remains therefore to show that $\mathfrak{D}_{A}=\mathfrak{D}_{A^{*}}\|A x\| \leq\left\|A^{*} x\right\|$ for each $x$ in $\mathfrak{D}_{A}$ and that there exists vector in $\mathfrak{D}_{A}$ for which $\|A x\|<\left\|A^{*} x\right\|$. We have

$$
\begin{aligned}
& \mathscr{D}_{A}=\left\{x=\left(\xi_{1}, \xi_{2} \ldots\right) ; \quad \sum_{i=2}^{\infty}(i-1)^{2}\left|\xi_{i}\right|^{2}<\infty\right\} \\
& \mathscr{D}_{A^{*}}=\left\{x=\left\{\left(\xi_{1}, \xi_{2} \ldots\right) ; \quad \sum_{i=1}^{\infty} i^{2}\left|\xi_{i}\right|^{2}<\infty\right\} .\right.
\end{aligned}
$$

For each $x \in \mathfrak{D}_{A^{*}} x \neq 0$ we have

$$
\|A x\|^{2}=\sum_{i=2}^{\infty}(i-1)^{2}\left|\xi_{i}\right|^{2}<\sum_{i=1}^{\infty} i^{2}\left|\xi_{i}\right|^{2}=\left\|A^{*} x\right\|^{2}
$$

Moreover, since $i^{2} \leq 2^{2}(i-1)^{2}$ for $i \geq 2$, the inequality

$$
\sum_{i=2}^{\infty}(i-1)^{2}\left|\xi_{i}\right|^{2}<\infty \text { implies } \sum_{i=1}^{\infty} i^{2}\left|\xi_{i}\right|^{2}<\infty .
$$

The semi-normality of $A$ is proved. 
3. - In the following Theorem 1 sp $A$ stands for «the spectrum of $A$ ».

Theorem 1. - Let $A$ be a closed operator whose adjoint is hyponormal, $B$ a bounded operator and $C$ such that

$$
A B-B A c C
$$

If sp $A$ is a non-trivial subset of the complex plane (i.e. it is not the whole plane and not the empty set) and if

$$
B\left(\mathfrak{D}_{A}\right) \subset \mathfrak{D}_{A}
$$

then

$$
\inf _{\substack{\|x\|=1 \\ x \in \mathfrak{D} C}}|(C x, x)|=0
$$

Proof. - Let $\mathfrak{B}$ be the complex plane. By assumption sp $A$ is a non-tri. vial closed subset of $B$. Let $\alpha$ be a point of the boundary of $\operatorname{sp} A$ and $\alpha_{n}$ a sequence in $\mathcal{B}-$ sp $A$ converging to $\alpha$. By (2) and (3)

$$
(A B-B A) x=C x
$$

for each $x$ in $\mathfrak{D}_{A}$. Hence

$$
\left(A-\alpha_{n} I\right) B x-B\left(A-\alpha_{n} I\right) x=C x
$$

for each $x$ in $\mathfrak{D}_{A}$ and for $n=1,2, \ldots$. Since $\alpha_{n} \in \mathfrak{B}$-sp $A, A-\alpha_{n} I$ has a bounded inverse $\left(A-\alpha_{n} I\right)^{-1}$. Let $y$ be a vector in $H$ with $\|y\|=1$. Since $A$ is closed and $A^{*}$ is hyponormal we have

$$
x=\left[\left(A-\alpha_{n} I\right)^{*}\right]^{-1} y \in \mathscr{D}_{A^{*}}=\mathscr{D}_{A^{* * *}}=\mathscr{D}_{A} .
$$

This vector may therefore be substituted in (4) and we get

$$
\begin{gathered}
\left.\left(A-\alpha_{n} I\right) B\left(\left(A-\alpha_{n} I\right)^{*}\right)\right)^{-1} y-B\left(A-\alpha_{n} I\left(\left(A-\alpha_{n} I\right)^{*}\right)^{-1} y=\right. \\
=C\left(\left(A-\alpha_{n} I\right)^{*}\right)^{-1} y .
\end{gathered}
$$

Applying $\left(A-\alpha_{n} I\right)^{-1}$ to both sides we obtain

$\left(A-\alpha_{n} I\right)^{-1} C\left(A-\alpha_{n} I\right)^{*} y=B\left(\left(A-\alpha_{n} I\right)^{*}\right)^{-1} y-\left(A-\alpha_{n} I\right)^{-1} B\left(A-\alpha_{n} I\right)\left(\left(A-\alpha_{n}\right)^{*}\right)^{-1} y$.

Since, as easily seen, hyponormality of $A^{*}$ implies hyponormality of $\left(A-\alpha_{n} I\right)^{*}$ it follows that

$$
\begin{gathered}
\left\|\left(A-\alpha_{n} I\right)^{-1} C\left(\left(A-\alpha_{n} I\right)^{*}\right)^{-1} y\right\| \leq\left\|B\left(\left(A-\alpha_{n} I\right)^{*}\right)^{-1} y\right\|+\left\|\left(A-\alpha_{n} I\right)^{-1} B\right\| \\
\left.\left\|\left(A-\alpha_{n} I\right)^{-1} C\left(\left(A-\alpha_{n} I\right)^{*}\right)^{-1} y\right\| \leq\|B\|\left\|\left(\left(A-\alpha_{n} I\right)^{*}\right)^{-1}\right\|\|y\|+\| A-\alpha_{n} I\right)^{-1}\|\| B\|\| y \| .
\end{gathered}
$$


Hence $\left(A-\alpha_{n} L\right)^{-1} C\left(\left(A-\alpha_{n} I\right)^{*}\right)^{-1}$ is bounded and

$$
\left\|\left(A-\alpha_{n} l\right)^{-1} C\left(\left(A-\alpha_{n} I\right)^{*}\right)^{-1}\right\| \leq 2\left\|\left(A-\alpha_{n} I\right)^{-1}\right\|\|B\| .
$$

On the other hand, for each $y,\|y\|=1, y \in H$

$$
\begin{aligned}
& \left\|\left(A-\alpha_{n} I\right)^{-1} C\left(\left(A-\alpha_{n} I\right)^{*}\right)^{-1} y\right\| \geq\left|\left(\left(A-\alpha_{n} I\right)^{-1} C\left(\left(A-\alpha_{n} I\right)^{*}\right)^{-1} y, y\right)\right|= \\
= & \mid\left(C\left(\left(A-\alpha_{n} I\right)^{*}\right)^{-1} y,\left(\left(A-\alpha_{n} I\right)^{*}\right)^{-1} y \mid=\frac{\left(C\left(\left(A-\alpha_{n} I\right)^{*-1} y,\left(\left(A-\alpha_{n} I\right)^{*}\right)^{-1} y\right)\right.}{\left\|\left(\left(A-\alpha_{n} I\right)^{*}\right)^{-1} y\right\|^{2}}\right. \\
& \left\|\left(\left(A-\alpha_{n} I\right)^{*}\right)^{-1} y\right\|^{2} .
\end{aligned}
$$

Hence

$$
\left\|\left(A-\alpha_{n} I\right)^{-1} C\left(\left(A-\alpha_{n} I\right)^{*}\right)^{-1} y\right\| \geq \operatorname{iuf}_{\substack{x \neq 0 \\ x \in D_{C}}} \frac{|(C x, x)|}{\|x\|^{2}}\left\|\left(\left(A-\alpha_{n} I\right)^{-1}\right)^{*} y\right\|^{2}
$$

and

$$
\begin{aligned}
& \left\|\left(A-\alpha_{n} I\right)^{-1} C\left(\left(A-\alpha_{n} I\right)^{*}\right)^{-1}\right\| \geq \inf _{\substack{\|x\|=1 \\
x \in \mathfrak{D} C}}|(C x, x)|\left\|\left(\left(A-\alpha_{n} I\right)^{-1}\right)^{*}\right\|^{2} \\
& =\inf _{\substack{\|x\| \|^{1} \\
x \in \mathfrak{D}_{C}}}|(C x, x)|\left\|\left(A-\alpha_{n}^{\sharp} I\right)^{-1}\right\|^{2} .
\end{aligned}
$$

Therefore, by (5) we have

$$
\inf _{\substack{\operatorname{lin}_{x \rightarrow=1} \| \in \mathbb{D} C \\ x \in \mathfrak{D} C}}|(C x, x)| \leq \frac{2\|B\|\left\|\left(A-\alpha_{n} I\right)^{-1}\right\|}{\left\|\left(A-\alpha_{n} l\right)^{-1}\right\|^{2}}=\frac{2 \mid B \|}{\left\|\left(A-\alpha_{n} I\right)^{-1}\right\|} .
$$

Since $\alpha_{n}$ is a sequence in $\mathfrak{B}_{-\mathrm{sp}} A$ coverging to $\alpha \in \mathrm{sp} A$ it follows that $\frac{1}{\alpha-\alpha_{n}} \in \operatorname{sp}\left(A-\alpha_{n} I\right)^{-1}$ (see $[1]$ p. 600). Hence $\frac{1}{|\alpha-\alpha|} \leq\left\|(A-\alpha I)^{-1}\right\|$.

This inequality and (6) imply

$$
\inf _{\substack{\|x\|=1 \\ x \in D_{C}}}|(C x, x)| \leq 2\|B\| \mid \alpha-\alpha_{n} !
$$

Since $\alpha_{n}-\alpha \rightarrow 0$ for $n \rightarrow \infty$ it follows that

$$
\inf _{\substack{\|x\|=1 \\ x \in \mathscr{D}_{C}}}|(C x, x)|=0
$$

Theorem 1 is proved. 
Theonem 2. - Let $A$ be a semi normal operator, $B$ a bounded operator and $C$ such that

$$
A B-B A c C \text {. }
$$

If sp $A$ is a non-trivial subset of the complex plane and the conditions

$$
\begin{gathered}
B\left(\mathfrak{D}_{A}\right) \subset \mathfrak{D}_{A} \\
B^{*}\left(\mathfrak{D}_{A}\right) \subset \mathfrak{D}_{A}
\end{gathered}
$$

are fulfilled, then

$$
\inf _{\substack{\|x\|=1 \\ x \in \mathfrak{D}_{C}}}|(C x, x)|=0
$$

Proof. - If $A^{*}$ is hyponormal then the theorem follows from theorem 1. We will suppose, therefore that $A$ is hyponormal. Let us define

Then

$$
C_{1}=A B-B A \quad D=A^{*} B^{*}-B^{*} A^{*}
$$

$$
C_{1} C \quad D_{\mathbf{c}}-C_{1}^{*}
$$

(see [4] p. 300]. Since (8) holds and $\mathscr{D}_{A}=\mathscr{D}_{A^{*}}$ we have by theorem 1

$$
\inf _{\substack{\| \prime x \\ x \in \mathfrak{D}_{A} \subset=\mathfrak{D}_{D}}}|(D x, x)|=0
$$

By (10) we get

$$
\inf _{\substack{\|x\|=1 \\ x \in \mathfrak{D}_{A}}}|(D x, x)|=\inf _{\substack{\| x \mid=1 \\ x \in \mathfrak{D}_{A}}}\left|\left(C_{1}^{*} x, x\right)\right|=\inf _{\substack{\|x\|=1 \\ x \in \mathfrak{D}_{A}}}\left|\left(C_{1} x, x\right)\right| \geq \inf _{\substack{\|x\|^{\prime} \\ x \in \mathfrak{D}_{C}}}|(C x, x)|
$$

and the theorem follows from (11).

RemarK 1. - It is easy to find examples of normal unbounded opera. tors $A$ and bounded operators $B$ satisfying the conditions of the theorem. Let as give now an example of an unbounded semi-normal not normal operator $A$ having a non trivial spectrum and a bounded operator $B$ satisfying the conditions (7) and (8). Let $H=K \oplus K$ where $K$ is a Hilbert Space.

Take

$$
A=\left(\begin{array}{ll}
P & 0 \\
O & Q
\end{array}\right) \quad B=\left(\begin{array}{ll}
M & N \\
S & T
\end{array}\right)
$$


where $P$ is a self adjoint unbounded operator in $K, Q$ a bounded seminormal operator on $K$ which is not normal, $M, N$ and $S$ are bounded operators on $K$ satisfying

$$
\begin{array}{cc}
M\left(\mathfrak{D}_{p}\right) \subset \mathfrak{D}_{p} & M^{*}\left(\mathfrak{D}_{p}\right) \subset \mathfrak{D}_{p} \\
\mathfrak{R}_{N} \subset \mathfrak{D}_{p} & \mathfrak{B}_{S^{*}} \subset \mathfrak{D}_{p}
\end{array}
$$

and $T$ is an arbitrary bounded operator on $K$. It is easily seen that the operator matrices $A$ and $B$ have the desired properties.

Remark 2. - Without the assumption that sp $A$ is a non-trivial subset of the complex plane Theorem 2 is not true. In fact, let $A$ be the semi-normal operator defined in (1) and $B=\left(b_{i j}\right)_{i, j=1}$ where $b_{j+1, j}=1$ for $j=1,2, \ldots$ and $b_{i j}=0$ for $i \neq j+1$.

It is easily seen that every complex number is an eigenvalue of $A$.

The conditions (7) and (8) are satisfied since

and

$$
\sum_{i=2}^{\infty} i^{2}\left|\xi_{i}\right|^{2} \leq \sum_{i=2}^{\infty} 2^{2}(i-1)^{2}\left|\xi_{i}\right|^{2}
$$

$$
\sum_{i=3}^{\infty}(i-2)^{2}\left|\xi_{i}\right|^{2}<\sum_{i=2}^{\infty}(i-1)^{2}\left|\xi_{i}\right|^{2}
$$

However, one may show easily that $A B-B A c I$ where 1 denotes the identity operator which does not satisfy (9).

Let us note that in the above remark $A$ is not a normal operator. In case that $A$ is normal we have

Theorem 3. - Let $A$ be a normal operator, $B$ a bounded operator and $C$ such that

$$
A B-B A c C \text {. }
$$

If

$$
B\left(\mathfrak{D}_{A}\right) \subset \mathfrak{D}_{A}
$$

then

$$
\inf _{\substack{\|x\|=1 \\ x \in \mathfrak{D} C}}|(C x, x)|=0
$$

Proof. - Since the spectrum of a normal operator is not empty the theorem follows from theorem 1 in case that sp $A$ is not the whole plane. 
Let us suppose that $\mathrm{sp} A$ is the whole plane. Let

$$
A=\int \lambda d E_{\lambda}
$$

be the spectral representation of $A$ and let $a_{n}$ be a sequence of positive numbers converging to zero. Define

$$
L_{n}=\left\{\lambda ; \lambda=c+i d, \quad \text { where } c, d \in\left[0, a_{n}\right]\right\}
$$

and

$$
\Phi_{n}(\lambda)= \begin{cases}\lambda & \lambda \in L_{n} \\ 0 & \lambda \notin L_{n}\end{cases}
$$

Let

$$
F_{n}=\int \Phi_{n}(\lambda) d E_{\lambda}
$$

and

$$
A_{n}=A-F_{n}=\int\left(\lambda-\Phi_{n}(\lambda)\right) d E_{\lambda} .
$$

Since $F_{n}$ is bounded $\mathscr{D}_{A x}=\mathscr{D}_{A}$ for $n=1,2, \ldots$. Therefore, (13) implies $B\left(\mathfrak{D}_{A n}\right) \subset \mathfrak{D}_{A n}$.

It is easily seen that $\mathrm{sp} A_{n}$ is a nontrivial subset of the plane for $n=1,2, \ldots$, Moreover, by (12) and (15) we have

$$
A_{n} B-B A_{n}=\left(A-F_{n}\right) B-B\left(A-F_{n}\right)=A B-B A+B F_{n}-F_{n} B \mathbf{c} C_{n}
$$

where

$$
C_{n}=C+B F_{n}-F_{n} B
$$

Therefore, $A_{n}, B$ and $C_{n}$ satisfy the conditions of theorem 1. Hence

for $n=1,2 \ldots$.

$$
\inf _{\substack{\|x\| \\ x \in \mathfrak{D} c_{n}}}\left|\left(C_{n} x, x\right)\right|=0
$$

Since $\left\|F_{n}\right\| \leq a_{n} \sqrt{ } 2 \rightarrow 0$ for $n \rightarrow \infty$ it follows that $B F_{n}-F_{n} B \rightarrow 0$ when $n \rightarrow \infty$. Thus, for each $x,\|x\|=1, x \in \mathscr{D}_{C_{n}}=\mathfrak{D}_{C}\left(C_{n} x, x\right) \rightarrow(C x, x)$ uniformly relative to $x$. Hence, by (16)

$$
\inf _{\substack{\|x\|=1 \\ x \in \mathfrak{D}_{C}}}|(C x, x)|=0
$$

Theorem 3 is proved. 
Note that if (13) is not satisfied Theorem 3 does not hold. This is shown by the following example:

Let $H=L_{2}[a, b], A=i \frac{d}{d x}, \mathscr{D}_{A}$ be the set of absolutely continuous complex functions $f$ in $[a, b]$ with $f^{\prime} \in L_{2}$ and $f(a)=f(b)$. Let $B=\Phi(x)$ where $\Phi(x)$ is any absolutely continuous complex function in $[\alpha, b]$ satisying

$$
\Phi(a) \neq \Phi(b) \quad \text { iRe } \Phi^{\prime}(x)>\alpha, \operatorname{Im} \Phi^{\prime}(x)>\beta
$$

for two positive numbers $\alpha$ and $\beta$.

Here $A$ is self adjoint and (12) holds for $C=i \Phi^{\prime}$ and $C$ does not satisfy (14). In this example (13) is not satisfied i.e. the equality

$$
(A B-B A) x=C x
$$

does not hold for each $x$ in $\mathscr{D}_{A}$.

However (17) holds on the set $\Omega$ of all functions of $\mathscr{D}_{A}$ vanishing at a and at $b, \Omega$ is obviously a dense subset of $H$. We also remark that $A(\Omega)$ is not dense. The following theorem (some ideas of which are similar to those of theorem 2.10.1 in [3]) shows that the fact that $A(\Omega)$ is not dense is not incidental.

Theonem 4. - Let $A$ be a self-adjoint operator, $B$ be a bounded operator and let $C$ be such that (17) holds on a linear subset $\Omega$ of $H$. If

$$
\inf _{\|x\|=1, x \in \mathfrak{D} C}|(C x, x)|=\alpha \neq 0
$$

Then $A(\Omega)$ is not dense in $H$.

Proof. - Suppose to the contrary that $A(\Omega)$ is dense in $H$. By (17) and (18) we have

$$
\begin{aligned}
\alpha\|x\|^{2} & \leq|(C x, x)|=|(A B x, x)-(B A x, x)| \leq|(B x, A x)|+\mid\left(A x, B^{*} x \mid \leq\right. \\
& \leq 2\|B\|\|A x\|\|x\|
\end{aligned}
$$

for each $x$ in $\Omega$. Hence

$$
\|x\| \leq \frac{2\|B\|}{\alpha}\|A x\|
$$

Let $y \in H$. Since $A(\Omega)$ is dense there exists a sequence $\left\{x_{n}\right\}$ in $A(\Omega)$ such that $A x_{n} \rightarrow y$. It follows, by (19) that there exists a vector $x$ in $H$ such that $x_{n} \rightarrow x$. Since $A$ is closed $x \in \mathscr{D}_{A}$ and $A x=y$. Hence $\mathfrak{R}_{A}=H$. Therefore, $A$ has a bounded self-adjoint inverse $A^{-1}$. 
Let $h \in A(\Omega)$. Putting in (17) $x=A^{-1} h$ we get

Thus

$$
A B A^{-1} h-B h=O A^{-1} h .
$$

$$
B A^{-1} h-A^{-1} B h=A^{-1} C A^{-1} h .
$$

Let $\mu \neq 0, \mu \in \mathrm{sp} A^{-1}$. Since $A^{-1}$ is bounded and self-adjoint there exists a sequence $y_{n}$ in $H$ with $\left\|y_{n}\right\|=1$ such that

$$
A^{-1} y_{n}-\mu y_{n} \rightarrow 0
$$

Let

$$
1>\varepsilon_{1}>\varepsilon_{2}>\varepsilon_{3}>\ldots
$$

be a sequence converging to zero. Since $A \Omega$ ) is dense there exists a sequence $\ell_{n}$ in $A(\Omega)$ such that

$$
\left\|t_{n}-y_{n}\right\|<\varepsilon_{n}
$$

Thus, by (21)

$$
A^{-1} t_{n}-\mu t_{n} \rightarrow 0
$$

Therefore by $\left\|y_{n}\right\|=1$ and by (22)

$$
\left\|t_{n}\right\|<1+\varepsilon_{n}<2
$$

and

$$
\left\|\ell_{n}\right\|>1-\varepsilon_{n}>1-\varepsilon_{1}>0 .
$$

By (24)

$$
\begin{aligned}
& \left|\left(B A^{-1} t_{n}, t_{n}\right)-\left(A^{-1} B t_{n}, t_{n}\right)\right|=\left|\left(B A^{-1} t_{n}, t_{n}\right)-\left(B t_{n}, A^{-1} t_{n}\right)\right|= \\
& =\mid\left(\left(B A^{-1}-\mu B\right) \ell_{n}, t_{n}-\left(B t_{n}, A^{-1} t_{n}-\mu t_{n}\right) \mid \leq\right. \\
& \left.\leq 2 \| B A^{-1}-\mu A\right) t_{n}\|+2\| B\|\| A^{-1} t_{n}-\mu t_{n} \| .
\end{aligned}
$$

By (23) both terms of the last expression tend to zero. As $n \rightarrow \infty$. Hence

$$
\left(B A^{-1} \ell_{n}, t_{n}\right)-\left(A^{-1} B t_{n}, t_{n}\right) \rightarrow 0
$$

as $n \rightarrow \infty$ and by $(20)$

$$
\left(O A^{-1} t_{n}, A^{-1} t_{n}\right)=\left(A^{-1} C A^{-1} t_{n}, t_{n}\right) \rightarrow 0 .
$$


But by (18) we have

$$
\alpha\left\|A^{-1} t_{n}\right\|^{2} \leq\left|\left(C A^{-1} t_{n}, A^{-1} t_{n}\right)\right|
$$

for $n=1,2 \ldots$. Hence $A^{-1} t_{n} \rightarrow 0$ and by (23) $t_{n} \rightarrow 0$. This contradicts (25). The theorem is proved.

Remark. - Theorem 4 is a generalization of theorem 2.10.1 in [3].

\section{REFERENCES}

[1] H. Dunford and J. Schwartz, Linear operators, Interscience, N.Y., Vol. 1 (1958).

[2] C. R. Putnam, on commutators of bounded matrices, Amer. Jour. Maih. (1951), pp. 127-131.

[3] - - Commutation properties of Hilbert Space operator's, Springer Verlag Berlin Heidelberg, New York (1967).

[4] F. Riesz and B.Sz. NagY, Functional Analysis, Frederick Ungar Pnb. Co. N.Y. (19āó). 